\title{
Counting the Number of Solutions to Certain Infinite Diophantine Equations
}

\author{
Nian Hong Zhou* and Yalin Sun
}

\begin{abstract}
Let $r, v, n$ be positive integers. This paper investigate the number of solutions $s_{r, v}(n)$ of the following infinite Diophantine equations

$$
n=1^{r} \cdot\left|k_{1}\right|^{v}+2^{r} \cdot\left|k_{2}\right|^{v}+3^{r} \cdot\left|k_{3}\right|^{v}+\cdots
$$

for $\boldsymbol{k}=\left(k_{1}, k_{2}, k_{3}, \ldots\right) \in \mathbb{Z}^{\infty}$. For each $(r, v) \in \mathbb{N} \times\{1,2\}$, a generating function and some asymptotic formulas of $s_{r, v}(n)$ are established.
\end{abstract}

\section{Introduction and statement of results}

Let $r, n$ be positive integers. A partition into $r$-th powers of an integer $n$ is a sequence of non-increasing $r$-th powers of positive integers whose sum equals $n$. Such a partition corresponds to a solution of the following infinite Diophantine equation:

$$
n=1^{r} \cdot k_{1}+2^{r} \cdot k_{2}+3^{r} \cdot k_{3}+\cdots
$$

for $\boldsymbol{k}=\left(k_{1}, k_{2}, k_{3}, \ldots\right) \in \mathbb{N}_{0}^{\infty}$. Let $p_{r}(n)$ be the number of partitions of $n$ into $r$-th powers and let $p_{r}(0):=1$, we have the generating function

$$
\sum_{n \geq 0} p_{r}(n) q^{n}=\prod_{n \geq 1} \frac{1}{1-q^{n^{r}}},
$$

where $q \in \mathbb{C}$ with $|q|<1$.

Determining the values of $p_{r}(n)$ has a long history and can be traced back to the work of Euler. In the famous paper [3], Hardy and Ramanujan proved an asymptotic expansion for $p_{1}(n)$ as $n \rightarrow \infty$. They [3, p. 111] also gave an asymptotic formula for $p_{r}(n), r \geq 2$, without proof. In 7, Theorem 2], Wright confirmed their asymptotic formula

$$
p_{r}(n) \sim \frac{c_{r} n^{\frac{1}{r+1}-\frac{3}{2}}}{\sqrt{(2 \pi)^{1+r}(1+1 / r)}} e^{(r+1) c_{r} n^{\frac{1}{r+1}}}
$$

as integer $n \rightarrow \infty$, where $c_{r}=\left(r^{-1} \zeta(1+1 / r) \Gamma(1+1 / r)\right)^{\frac{r}{r+1}}, \zeta(\cdot)$ is the Riemann zeta function and $\Gamma(\cdot)$ is the classical Euler Gamma function.

Received May 15, 2020; Accepted November 29, 2020.

Communicated by Yifan Yang.

2020 Mathematics Subject Classification. 11D45, 11P82, 11P99.

Key words and phrases. integer partitions, infinite Diophantine equations, asymptotics.

*Corresponding author. 
In this paper we investigate certain infinite Diophantine equation analogous to (1.1). For given positive integers $n$ and $r$, we use $s_{r, v}(n)$ to denote the number of solutions of the following infinite Diophantine equation

$$
n=1^{r} \cdot\left|k_{1}\right|^{v}+2^{r} \cdot\left|k_{2}\right|^{v}+3^{r} \cdot\left|k_{3}\right|^{v}+\cdots
$$

for $\boldsymbol{k}=\left(k_{1}, k_{2}, k_{3}, \ldots\right) \in \mathbb{Z}^{\infty}$. The first result of this paper is about the generating function for $s_{r, v}(n)$.

Proposition 1.1. Let $s_{r, v}(0):=1$ and $q \in \mathbb{C}$ with $|q|<1$. We have

$$
G_{r, 1}(q):=\sum_{n \geq 0} s_{r, 1}(n) q^{n}=\prod_{n \geq 1} \frac{1+q^{n^{r}}}{1-q^{n^{r}}}
$$

and

$$
G_{r, 2}(q):=\sum_{n \geq 0} s_{r, 2}(n) q^{n}=\prod_{j \geq 1} \prod_{n \geq 1} \frac{1-(-1)^{n} q^{n j^{r}}}{1+(-1)^{n} q^{n j^{r}}} .
$$

Remark 1.2. From the proof of this proposition (see Subsection 2.1), the above infinite product expansion for $G_{r, s}(q)(r \in \mathbb{N}, s=1,2)$ follows the identities

$$
\sum_{n \in \mathbb{Z}} q^{|n|}=\frac{1+q}{1-q} \text { and } \sum_{n \in \mathbb{Z}} q^{|n|^{2}}=\prod_{n \geq 1} \frac{1-(-q)^{n}}{1+(-q)^{n}}
$$

They actually follow from the geometric sequence sum formula and the Jacobi triple product identity. However, any useful expansion for the sum $\sum_{n \in \mathbb{Z}} q^{|n|^{v}}$ with each integer $v>2$ is still not found yet. Therefore, whether there are infinite product formulas which is similar to Proposition 1.1 for $s_{r, v}(n)\left(r \in \mathbb{N}, v \in \mathbb{Z}_{>2}\right)$ is still a question to be settled.

Thanks to the infinite product expansion in Proposition 1.1, we can determine the asymptotic behavior of $G_{r, v}(q)$ when $|q| \rightarrow 1^{-}$. From which we can further determine the asymptotics of $s_{r, v}(n)((r, v) \in \mathbb{N} \times\{1,2\})$ as $n \rightarrow \infty$. More precisely, we prove

Theorem 1.3. For any given positive integers $r$ and $p$, we have

$$
s_{r, 1}(n)=\frac{\kappa_{r}^{3 / 2}}{\sqrt{2^{r+1} \pi^{r}}}\left(\frac{1}{n}\right)^{\frac{1+1 / 2}{1+1 / r}} W_{\frac{1}{r}, \frac{1}{2}}\left(\kappa_{r} n^{\frac{1}{1+r}}\right)\left(1+O\left(\frac{1}{n^{p}}\right)\right)
$$

and

$$
s_{r, 2}(n)=\frac{\kappa_{r}^{5 / 4}}{\sqrt[4]{2^{r} \pi^{r+1}}}\left(\frac{\eta(1 / r)}{n}\right)^{\frac{1+1 / 4}{1+1 / r}} W_{\frac{1}{r}, \frac{1}{4}}\left(\kappa_{r} \eta(1 / r)\left(\frac{n}{\eta(1 / r)}\right)^{\frac{1}{1+r}}\right)\left(1+O\left(\frac{1}{n^{p}}\right)\right)
$$

as integer $n \rightarrow \infty$. Here $\kappa_{r}>0$ is given by

$$
\kappa_{r}^{1+1 / r}=2 r^{-1}\left(1-2^{-1-1 / r}\right) \zeta(1+1 / r) \Gamma(1+1 / r),
$$


$\eta(s)=\sum_{n \geq 1}(-1)^{n-1} n^{-s}$ is the Dirichlet eta function, and

$$
W_{\alpha, \beta}(\lambda)=\frac{1}{2 \pi} \int_{-1}^{1}(1+\mathrm{i} u)^{\beta} \exp \left(\lambda\left(\alpha^{-1}(1+\mathrm{i} u)^{-\alpha}+(1+\mathrm{i} u)\right)\right) d u
$$

for all $\alpha, \beta, \lambda>0$.

Using the standard saddle-point method, such as referring to [5, p. 127, Theorem 7.1], we can derive an asymptotic expansion for $W_{\alpha, \beta}(\lambda)$ as $\lambda \rightarrow+\infty$. Hence it is possible to derive full asymptotic expansions for $s_{r, v}(n)((r, v) \in \mathbb{N} \times\{1,2\})$. In particular, we have the following leading asymptotics.

Corollary 1.4. For any given positive integer $r$, we have

$$
s_{r, 1}(n) \sim 2^{-(r+2) / 2} \pi^{-(r+1) / 2}(1+1 / r)^{-1 / 2} \kappa_{r} n^{-\frac{3 r+1}{2+2 r}} e^{(1+r) \kappa_{r} n^{\frac{1}{1+r}}}
$$

and

$$
s_{r, 2}(n) \sim 2^{-(r+2) / 4} \pi^{-(r+3) / 4}(1+1 / r)^{-1 / 2} \eta(1 / r)^{\frac{3 r}{4 r+4}} \kappa_{r}^{3 / 4} n^{-\frac{5 r+2}{4+4 r}} e^{(1+r) \kappa_{r} \eta(1 / r)^{\frac{r}{1+r}}} n^{\frac{1}{1+r}}
$$

as $n \rightarrow \infty$.

2. Some results of the generating function

\subsection{Proof of Proposition 1.1}

We shall proceed in a formal manner to prove Proposition 1.1 . Formally, using 1.2 we have

$$
\begin{aligned}
\sum_{n \geq 0} s_{r, v}(n) q^{n} & =\sum_{n \geq 0} q^{n} \sum_{\substack{k \in \mathbb{Z}^{\infty} \\
\sum_{j \geq 1} j^{r}\left|k_{j}\right|^{s}=n}} 1 \\
& =\sum_{\boldsymbol{k} \in \mathbb{Z}^{\infty}} q^{\sum_{j \geq 1} j^{r}\left|k_{j}\right|^{s}}=\prod_{j \geq 1}\left(\sum_{k_{j} \in \mathbb{Z}} q^{j^{r}\left|k_{j}\right|^{s}}\right) .
\end{aligned}
$$

Now, for $q \in \mathbb{C}$ with $|q|<1$, by noting that

$$
\sum_{n \in \mathbb{Z}} q^{|n|}=1+2 \sum_{n \geq 1} q^{n}=\frac{1+q}{1-q}
$$

and an identity of Gauss (see Andrews [1, Corollary 2.10])

$$
\sum_{n \in \mathbb{Z}} q^{n^{2}}=\prod_{n \geq 1} \frac{1-(-q)^{n}}{1+(-q)^{n}}
$$


we have

$$
G_{r, 1}(q):=\sum_{n \geq 0} s_{r, 1}(n) q^{n}=\prod_{n \geq 1} \frac{1+q^{n^{r}}}{1-q^{n^{r}}}
$$

and

$$
G_{r, 2}(q):=\sum_{n \geq 0} s_{r, 2}(n) q^{n}=\prod_{j \geq 1} \prod_{n \geq 1} \frac{1-(-1)^{n} q^{n j^{r}}}{1+(-1)^{n} q^{n j^{r}}} .
$$

Clearly, the product for $G_{r, 1}(q)$ is absolute convergence for all $q \in \mathbb{C}$ with $|q|<1$. For the product for $G_{r, 2}(q)$, since

$$
\left|\prod_{j \geq 1} \prod_{n \geq 1} \frac{1-(-1)^{n} q^{n j^{r}}}{1+(-1)^{n} q^{n j^{r}}}\right| \leq \prod_{j \geq 1} \prod_{n \geq 1} \frac{1+|q|^{n j^{r}}}{1-|q|^{n j^{r}}}=\prod_{\ell \geq 1}\left(\frac{1+|q|^{\ell}}{1-|q|^{\ell}}\right)^{\sigma_{1, r}(\ell)}
$$

where

$$
\sigma_{1, r}(\ell)=\#\left\{(n, j) \in \mathbb{N}^{2}: n j^{r}=\ell\right\} \leq \ell ;
$$

and hence the product is absolute convergence for all $q \in \mathbb{C}$ with $|q|<1$. This completes the proof of Proposition 1.1 .

\subsection{Asymptotics of the generating function}

To give a proof of Theorem 1.3, we need to determine asymptotics of the generating function in Proposition 1.1 at $q=1$.

Proposition 2.1. Let $r$ be a given positive integer, $z=x+\mathrm{i} y$ with $x, y \in \mathbb{R}$ and $|\arg (z)| \leq$ $\pi / 4$. As $z \rightarrow 0$,

$$
G_{r, 1}\left(e^{-z}\right)=\frac{z^{1 / 2} \exp \left(r \kappa_{r}^{1+1 / r} z^{-1 / r}\right)}{\sqrt{2^{r+1} \pi^{r}}}\left(1+O\left(|z|^{p}\right)\right)
$$

and

$$
G_{r, 2}\left(e^{-z}\right)=\frac{z^{1 / 4} \exp \left(r \eta(1 / r) \kappa_{r}^{1+1 / r} z^{-1 / r}\right)}{\sqrt[4]{2^{r} \pi^{r+1}}}\left(1+O\left(|z|^{p}\right)\right)
$$

holds for any given $p>0$. Here $\kappa_{r}>0$ such that

$$
\kappa_{r}^{1+1 / r}=2 r^{-2}\left(1-2^{-1-1 / r}\right) \zeta(1+1 / r) \Gamma(1 / r) .
$$

Proof. The proof of the result for $G_{r, 1}\left(e^{-z}\right)$ is similar to $G_{r, 2}\left(e^{-z}\right)$, hence we only prove the later one. We shall follow the proof of [1, p. 89, Lemma 6.1]. The series for the Riemann zeta function

$$
\zeta(s)=\sum_{n \geq 1} n^{-s}
$$


and the Dirichlet eta function

$$
\eta(s)=\sum_{n \geq 1}(-1)^{n-1} n^{-s}
$$

converge absolutely and uniformly for $s \in \mathbb{C}$ when $\Re(s) \geq c>1$. Therefore, by using Mellin's transform,

$$
\begin{aligned}
\log G_{r, 2}\left(e^{-z}\right) & =2 \sum_{\substack{\ell \geq 1 \\
\ell \text { odd }}} \frac{1}{\ell} \sum_{j \geq 1} \sum_{n \geq 1}(-1)^{n-1} e^{-n \ell j^{r} z} \\
& =2 \sum_{\substack{\ell \geq 1 \\
\ell \text { odd }}} \frac{1}{\ell} \sum_{j \geq 1} \sum_{n \geq 1}(-1)^{n-1} \frac{1}{2 \pi \mathrm{i}} \int_{c-\mathrm{i} \infty}^{c+\mathrm{i} \infty}\left(n \ell j^{r} z\right)^{-s} \Gamma(s) d s \\
& =\frac{2}{2 \pi \mathrm{i}} \int_{c-\mathrm{i} \infty}^{c+\mathrm{i} \infty}\left(\sum_{\substack{\ell \geq 1 \\
\ell \text { odd }}} \frac{1}{\ell^{s+1}} \sum_{n \geq 1} \frac{(-1)^{n-1}}{n^{s}} \sum_{j \geq 1} \frac{1}{j^{r s}}\right) \Gamma(s) z^{-s} d s,
\end{aligned}
$$

that is

$$
\log G_{r, 2}\left(e^{-z}\right)=\frac{2}{2 \pi \mathrm{i}} \int_{c-\mathrm{i} \infty}^{c+\mathrm{i} \infty}\left(1-2^{-1-s}\right) \zeta(s+1) \eta(s) \zeta(r s) \Gamma(s) z^{-s} d s
$$

for all $z \in \mathbb{C}$ with $\Re(z)>0$. Since the only poles of gamma function $\Gamma(s)$ are at $s=-k$ $\left(k \in \mathbb{Z}_{\geq 0}\right)$, and all are simple; $\eta(s)$ is an entire function on $\mathbb{C}$; all $s=-2 k(k \in \mathbb{N})$ are zeros of zeta function $\zeta(s)$, and $s=1$ is the only pole of $\zeta(s)$ and is simple. Thus, it is easy to check that the only possible poles of the integrand

$$
g_{r}(s) z^{-s}:=\left(1-2^{-1-s}\right) \zeta(s+1) \eta(s) \zeta(r s) \Gamma(s) z^{-s}
$$

are at $s=0$ and $1 / r$. For all $\sigma \in[a, b], a, b \in \mathbb{R}$ and real number $t,|t| \geq 1$, we have the well-known classical facts (see [6, p. 38, p. 92]) that

$$
\Gamma(\sigma+\mathrm{i} t) \ll_{a, b}|t|^{\sigma-1 / 2} \exp \left(-\frac{\pi}{2}|t|\right) \quad \text { and } \quad \zeta(\sigma+\mathrm{i} t) \ll_{a, b}|t|^{|\sigma|+1 / 2} .
$$

Hence we have $g_{r}(s) \ll_{a, b}|t|^{O(1)} \exp \left(-\frac{\pi}{2}|t|\right)$. Thus, using the residue theorem, moving the line of integration (2.1) to the $\Re(s)=-p$ with any given $p>0$, and taking into account the possible pole at $s=0$ and $s=1 / r$ of $g(s)$, we obtain

$$
\log G_{r, 2}\left(e^{-z}\right)=2 \sum_{s \in\{0,1 / r\}} \operatorname{Res}\left(g_{r}(s) z^{-s}\right)+O\left(|z|^{p}\right)
$$

as $z \rightarrow 0$ with $|\arg (z)|<\pi / 4$. By Laurent expansion of $\zeta(s+1)$ and $\Gamma(s)$ at $s=0$, we have

$$
\zeta(s+1)=1 / s+\gamma+O(|s|) \quad \text { and } \quad \Gamma(s)=1 / s-\gamma+O(|s|)
$$


as $s \rightarrow 0$. Therefore,

$$
\operatorname{Res}_{s=1 / r}\left(g_{r}(s) z^{-s}\right)=\frac{\left(1-2^{-1-1 / r}\right) \zeta(1 / r+1) \eta(1 / r) \Gamma(1 / r)}{r z^{1 / r}}
$$

and

$$
\operatorname{Res}_{s=0}\left(g_{r}(s) z^{-s}\right)=\frac{1}{8} \log \left(\frac{z}{2^{r} \pi^{r+1}}\right) .
$$

Combining $(2.2)$ with above results, we obtain the proof of this proposition.

We also need the following upper bound results.

Lemma 2.2. Let $(r, v) \in \mathbb{N} \times\{1,2\}$ be given, $z=x+\mathrm{i} y$ with $x \in \mathbb{R}_{+}$and $y \in(-\pi, \pi] \backslash$ $(-x, x)$. As $x \rightarrow 0$,

$$
\Re\left(\log \frac{G_{r, v}\left(e^{-x}\right)}{G_{r, v}\left(e^{-z}\right)}\right) \gg x^{-1 / r} .
$$

Proof. By using Proposition 1.1 with $q \in \mathbb{C}$ and $|q|<1$, we have

$$
\begin{aligned}
\log G_{r, 1}(q) & =\sum_{j \geq 1} \log \left(\frac{1+q^{j^{r}}}{1-q^{j^{r}}}\right) \\
& =\sum_{j \geq 1}\left(\sum_{\ell \geq 1}(-1)^{\ell-1} \frac{q^{\ell j^{r}}}{\ell}+\sum_{\ell \geq 1} \frac{q^{\ell j^{r}}}{\ell}\right) \\
& =\sum_{\ell \geq 1} \frac{1}{\ell} \sum_{j \geq 1}\left((-1)^{\ell-1}+1\right) q^{\ell j^{r}}=2 \sum_{\substack{\ell \geq 1 \\
\ell \text { odd }}} \frac{1}{\ell \geq 1} \sum_{j} q^{j^{r} \ell}
\end{aligned}
$$

and

$$
\begin{aligned}
\log G_{r, 2}(q) & =\sum_{n, j \geq 1} \log \left(\frac{1-(-1)^{n} q^{n j^{r}}}{1+(-1)^{n} q^{n j^{r}}}\right) \\
& =\sum_{n, j \geq 1} \sum_{\ell \geq 1} \frac{1}{\ell}\left(-(-1)^{n \ell} q^{n j^{r} \ell}+(-1)^{\ell}(-1)^{n \ell} q^{n j^{r} \ell}\right) \\
& =\sum_{\ell, j \geq 1} \frac{(-1)^{\ell}-1}{\ell} \frac{\left(-q^{j^{r}}\right)^{\ell}}{1-\left(-q^{j^{r}}\right)^{\ell}}=2 \sum_{\substack{\ell \geq 1 \\
\ell \text { odd }}} \frac{1}{\ell} \sum_{j \geq 1} \frac{q^{j^{r} \ell}}{1+q^{j^{r} \ell}} .
\end{aligned}
$$

Furthermore,

$$
\Re\left(\log \frac{G_{r, 1}\left(e^{-x}\right)}{G_{r, 1}\left(e^{-z}\right)}\right)=2 \sum_{\substack{\ell \geq 1 \\ \ell \text { odd }}} \frac{1}{\ell} \sum_{j \geq 1} e^{-j^{r} \ell x} \Re\left(1-\exp \left(2 \pi \mathrm{i} \ell j^{r} \frac{y}{2 \pi}\right)\right)
$$


and

$$
\begin{aligned}
\Re\left(\log \frac{G_{r, 2}\left(e^{-x}\right)}{G_{r, 2}\left(e^{-z}\right)}\right) & =2 \sum_{\substack{\ell \geq 1 \\
\ell \text { odd }}} \frac{1}{\ell} \sum_{j \geq 1} \Re\left(\frac{e^{-j^{r} \ell x}}{1+e^{-j^{r} \ell x}}-\frac{e^{-j^{r} \ell z}}{1+e^{-j^{r} \ell z}}\right) \\
& =2 \sum_{\substack{\ell \geq 1 \\
\ell \text { odd }}} \frac{1}{\ell} \sum_{j \geq 1} \frac{\tanh \left(j^{r} \ell \frac{x}{2}\right)}{\cosh \left(j^{r} \ell x\right)+\cos \left(j^{r} \ell y\right)} \sin ^{2}\left(j^{r} \ell \frac{y}{2}\right) .
\end{aligned}
$$

By noting that all summand in above sums are nonnegative we have

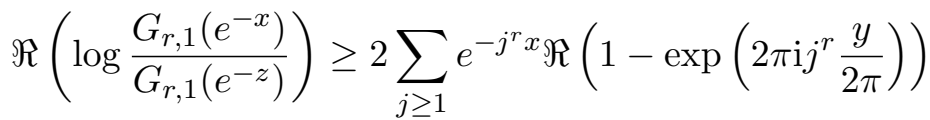

$$
\begin{aligned}
& \gg \sum_{(2 \pi / x)^{1 / r}<j \leq 2(2 \pi / x)^{1 / r}} \Re\left(1-\exp \left(2 \pi \mathrm{i} j^{r} \frac{y}{2 \pi}\right)\right)
\end{aligned}
$$

and

$$
\begin{aligned}
\Re\left(\log \frac{G_{r, 2}\left(e^{-x}\right)}{G_{r, 2}\left(e^{-z}\right)}\right) & \geq 2 \sum_{j \geq 1} \frac{\tanh \left(j^{r} \frac{x}{2}\right)}{\cosh \left(j^{r} x\right)+\cos \left(j^{r} y\right)} \sin ^{2}\left(j^{r} \frac{y}{2}\right) \\
& \gg \sum_{(2 \pi / x)^{1 / r}<j \leq 2(2 \pi / x)^{1 / r}} \Re\left(1-\exp \left(2 \pi \mathrm{i} j^{r} \frac{y}{2 \pi}\right)\right) .
\end{aligned}
$$

Thus by using Lemma 2.3 with $L=(2 \pi / x)^{1 / r}$, we find that

$$
\Re\left(\log \frac{G_{r, v}\left(e^{-x}\right)}{G_{r, v}\left(e^{-z}\right)}\right) \gg \delta_{r}(2 \pi / x)^{1 / r} \gg x^{-1 / r}
$$

holds for all sufficiently small $x>0$ and $v \in\{1,2\}$. This finishes the proof.

Lemma 2.3. Let $r \in \mathbb{N}, y \in \mathbb{R}$ and $L \in \mathbb{R}_{+}$such that $L^{-r}<|y| \leq 1 / 2$. Then there exists a constant $\delta_{r} \in(0,1)$ depending only on $r$ such that

$$
\left|\sum_{L<n \leq 2 L} e^{2 \pi \mathrm{i} n^{r} y}\right| \leq\left(1-\delta_{r}\right) L
$$

holds for all positive sufficiently large $L$.

Proof. The lemma for $r=1$ is easy and we shall focus on the cases of $r \geq 2$. By the well-known Dirichlet's approximation theorem, for any $y \in \mathbb{R}$ and $L>0$ being sufficiently large, then there exist integers $d$ and $h$ with $0<h \leq L^{r-1}$ and $\operatorname{gcd}(h, d)=1$ such that

$$
\left|y-\frac{d}{h}\right|<\frac{1}{h L^{r-1}}
$$


The use of [4, Equation 20.32] implies that

$$
\sum_{L<n \leq 2 L} e^{2 \pi \mathrm{i} n^{r} y}=\frac{1}{h} \sum_{1 \leq j \leq h} e^{2 \pi \mathrm{i} j^{r} \frac{d}{h}} \int_{L}^{2 L} e^{2 \pi \mathrm{i} u^{r}\left(y-\frac{d}{h}\right)} d u+O(h) .
$$

If the real number $y$ satisfies $L^{-r}<|y| \leq L^{1-r}$, then $y$ satisfies the approximation (2.3) with $(h, d)=(1,0)$. This means that

$$
\begin{aligned}
\left|\sum_{L<n \leq 2 L} e^{2 \pi \mathrm{i} n^{r} y}\right| & =\left|\int_{L}^{2 L} e^{2 \pi \mathrm{i} u^{r} y} d u+O(1)\right| \\
& \leq 2 \cdot \frac{1}{2 \pi r|y| L^{r-1}}\left(1+2^{1-r}\right)+O(1) \leq \frac{1+2^{1-r}}{\pi r} L+O(1) .
\end{aligned}
$$

If the real number $y$ satisfies $1 / 2 \geq|y| \geq L^{1-r}$, then $y$ satisfies the approximation (2.3) with $h \geq 2$. Further, by using [2, Lemma 2.1] in (2.4), we find that there exists a positive constant $\delta_{r 1}$ depending only on $r$ such that

$$
\left|\sum_{L<n \leq 2 L} e^{2 \pi \mathrm{i} n^{r} y}\right| \leq\left(1-\delta_{r 1}\right) L+O(h) .
$$

On the other hand, the use of Weyl's inequality (see [4, Lemma 20.3]) implies that

$$
\sum_{L<j \leq 2 L} e^{2 \pi \mathrm{i} j^{r} y} \ll_{\varepsilon} L^{1+\varepsilon}\left(h^{-1}+L^{-1}+h L^{-r}\right)^{2^{1-r}} \ll L^{1-2^{-r-1 / 2}}
$$

holds for all integers $h \in\left(L^{1 / 2}, L^{r-1}\right]$. By using (2.5), (2.6) and (2.7), it is not difficult to obtain the proof of the lemma.

\section{Proof of the main theorem}

From Proposition 2.1 and Lemma 2.2, we can check that the sequences $\left\{s_{r, 1}(n)\right\}_{n \geq 0}$ and $\left\{s_{r, 2}(n)\right\}_{n \geq 0}$ satisfy the conditions of Proposition 3.1 below. Therefore, applying the following proposition, Theorem 1.3 and Corollary 1.4 follow.

Proposition 3.1. For a sequence $\left\{c_{n}\right\}_{n \geq 0}$ of real numbers, we let $G(q):=\sum_{n \geq 0} c_{n} q^{n}$. Suppose that for $x \in \mathbb{R}_{+}$and $y \in(-\pi, \pi]$,

$$
G\left(e^{-x-\mathrm{i} y}\right)-\gamma(x+\mathrm{i} y)^{\beta} e^{\kappa \alpha^{-1}(x+\mathrm{i} y)^{-\alpha}} \ll x^{p} G\left(e^{-x}\right), \quad x \rightarrow 0
$$

holds for any given $p>0$, where $\kappa, \gamma, \beta, \alpha \in \mathbb{R}_{+}$. Then, for any given $p>0$ we have

$$
c_{n}=\gamma\left(\frac{\kappa}{n}\right)^{\frac{1+\beta}{1+\alpha}} W_{\alpha, \beta}\left(\kappa^{\frac{1}{1+\alpha}} n^{\frac{\alpha}{1+\alpha}}\right)\left(1+O\left(n^{-p}\right)\right)
$$

as integer $n \rightarrow \infty$. In particular,

$$
c_{n} \sim 2^{-1 / 2} \pi^{-1 / 2}(1+\alpha)^{-1 / 2} \gamma \kappa^{\frac{\beta+1 / 2}{1+\alpha}} n^{-\frac{1+\beta+\alpha / 2}{1+\alpha}} e^{\left(1+\alpha^{-1}\right) \kappa^{\frac{1}{1+\alpha}} n^{\frac{\alpha}{1+\alpha}}}, \quad n \rightarrow \infty .
$$


Proof. For any given positive integer $n$ sufficiently large, by using the orthogonality we have

$$
c_{n}=\frac{1}{2 \pi} \int_{-\pi}^{\pi} G\left(e^{-x-\mathrm{i} y}\right) e^{n x+n \mathrm{i} y} d y .
$$

We split the above integral as

$$
\begin{aligned}
c_{n}= & \frac{1}{2 \pi} \int_{-x}^{x} \gamma(x+\mathrm{i} y)^{\beta} e^{\kappa \alpha^{-1}(x+\mathrm{i} y)^{-\alpha}+n(x+\mathrm{i} y)} d y \\
& +\frac{1}{2 \pi} \int_{-\pi}^{\pi}\left(G\left(e^{-x-\mathrm{i} y}\right)-\gamma(x+\mathrm{i} y)^{\beta} e^{\kappa \alpha^{-1}(x+\mathrm{i} y)^{-\alpha}}\right) e^{n(x+\mathrm{i} y)} d y \\
= & : I(n)+E(n) .
\end{aligned}
$$

Let $x=\left(\frac{\kappa}{n}\right)^{\frac{1}{\alpha+1}}$. For $E(n)$, we estimate that

$$
\begin{aligned}
E(n) & \ll \int_{-\pi}^{\pi} x^{(1+\alpha) p} G\left(e^{-x}\right) e^{n x} d y \\
& \ll \int_{-\pi}^{\pi} x^{p} e^{\kappa \alpha^{-1} x^{-\alpha}+n x} d y \ll n^{-p} e^{\left(\alpha^{-1}+1\right) \kappa^{\frac{1}{1+\alpha}} n^{\frac{\alpha}{1+\alpha}}}
\end{aligned}
$$

holds for any given $p>0$. For $I(n)$, we compute that

$$
\begin{aligned}
I(n) & =\frac{\gamma}{2 \pi \mathrm{i}} \int_{x-\mathrm{i} x}^{x+\mathrm{i} x} z^{\beta} e^{\kappa \alpha^{-1} z^{-\alpha}+n z} d z \\
& =\frac{\gamma x^{1+\beta}}{2 \pi \mathrm{i}} \int_{1-\mathrm{i}}^{1+\mathrm{i}} u^{\beta} e^{\kappa \alpha^{-1} x^{-\alpha} u^{-\alpha}+n x u} d u \\
& =\gamma\left(\frac{\kappa}{n}\right)^{\frac{1+\beta}{1+\alpha}} \frac{1}{2 \pi \mathrm{i}} \int_{1-\mathrm{i}}^{1+\mathrm{i}} u^{\beta} e^{\kappa^{\frac{1}{1+\alpha}} n^{\frac{\alpha}{1+\alpha}}\left(\alpha^{-1} u^{-\alpha}+u\right)} d u
\end{aligned}
$$

that is

$$
I(n)=\gamma\left(\frac{\kappa}{n}\right)^{\frac{1+\beta}{1+\alpha}} W_{\alpha, \beta}\left(\kappa^{\frac{1}{1+\alpha}} n^{\frac{\alpha}{1+\alpha}}\right) .
$$

By using the standard Laplace saddle-point method (see, for example, [5, p. 127, Theorem 7.1]), since the integral

$$
W_{\alpha, \beta}(\lambda)=\frac{1}{2 \pi} \int_{-1}^{1}(1+\mathrm{i} u)^{\beta} \exp \left(\lambda\left(\alpha^{-1}(1+\mathrm{i} u)^{-\alpha}+(1+\mathrm{i} u)\right)\right) d u
$$

has a simple saddle point $u=0$, it is not difficult to prove that

$$
W_{\alpha, \beta}(\lambda) \sim \frac{1}{\sqrt{2 \pi(1+\alpha)}} \frac{e^{\left(1+\alpha^{-1}\right) \lambda}}{\lambda^{1 / 2}}
$$

as $\lambda \rightarrow+\infty$. The proof of Proposition 3.1 follows from $(3.1)-(3.3)$ and $(3.4)$. This completes the proof. 


\section{Acknowledgments}

This research was partly supported by the National Science Foundation of China (Grant No. 11971173). The authors would like to thank the anonymous referee for his/her very helpful comments and suggestions.

\section{References}

[1] G. E. Andrews, The Theory of Partitions, Encyclopedia of Mathematics and its Applications 2, Addison-Wesley, Reading, Mass.-London-Amsterdam, 1976.

[2] A. Gafni, Power partitions, J. Number Theory 163 (2016), 19-42.

[3] G. H. Hardy and S. Ramanujan, Asymptotic formulaae in combinatory analysis, Proc. London Math. Soc. (2) 17 (1918), 75-115.

[4] H. Iwaniec and E. Kowalski, Analytic Number Theory, American Mathematical Society Colloquium Publications 53, American Mathematical Society, Providence, RI, 2004.

[5] F. W. J. Olver, Asymptotics and Special Functions, Computer Science and Applied Mathematics, Academic Press, New York-London, 1974.

[6] H. Rademacher, Topics in Analytic Number Theory, Die Grundlehren der mathematischen Wissenschaften, Band 169, Springer-Verlag, New York-Heidelberg, 1973.

[7] E. M. Wright, Asymptotic partition formulae III: Partitions into $k$-th powers, Acta Math. 63 (1934), no. 1, 143-191.

Nian Hong Zhou

School of Mathematics and Statistics, Guangxi Normal University, No. 1 Yanzhong Road, Yanshan District, 541006 Guilin, Guangxi, China

and

School of Mathematical Sciences, East China Normal University, 500 Dongchuan Road, Minhang District, 200241 Shanghai, China

E-mail addresses: nianhongzhou@outlook.com, nianhongzhou@gxnu.edu.cn

\section{Yalin Sun}

School of Mathematical Sciences, East China Normal University, 500 Dongchuan Road, Shanghai 200241, China

E-mail address: yalinsun@outlook.com 